\title{
Using Carbon-fibre Reinforcement with a 5-axis Material Extrusion System
}

\author{
Nathaniel Kaill ${ }^{1, *}$, Patrick Pradel $^{1}$, Guy Bingham $^{2}$ and $R$ Ian Campbell ${ }^{1}$ \\ ${ }^{1}$ Loughborough Design School, Loughborough University, LE11 3TU, UK \\ ${ }^{2}$ Institute of Art and Design, De Montfort University, LE1 9BH, UK
}

\begin{abstract}
One of the main limitations of material extrusion (ME) components is their anisotropic mechanical behaviour, mainly due to the poor bonding between layers. 5-axis ME has the capability to orientate the print layers in line with loading conditions, in order to limit the effect of poor inter-laminar bonding. Previous work has demonstrated how aligning deposited material in the same direction as the dominant stresses can improve a part's mechanical performance. When fibre-reinforcement is added to these oriented layers, the stiffness and strength of parts should increase further. This paper presents a comparison between 5 -axis parts that were printed in pure poly-lactic acid (PLA) and in carbon-fibrereinforced (CFR) PLA. Sets of dome-shaped components were built using several different build strategies and tested for compressive stiffness and strength. The results were rather mixed but did show a marked improvement in compressive strength under certain conditions. Further work is required to understand one of the failure mechanisms that was seen and to overcome some of the limitations of the 5-axis machine currently being used. The work was undertaken to support the Directional Composites through Innovative Manufacturing (DiCoMI) project, funded by the European Commission.
\end{abstract}

\section{Introduction}

Most material extrusion (ME) systems used for additive manufacturing (AM) work in one material only or in a combination of discrete materials, e.g. one material as the build material and a second as the support material. Many ME systems are capable of using composite materials for aesthetic purposes, e.g. polymer loaded with metal particles or wood fibres to give a metallic or wooden appearance (see Figure 1). A few commercial ME systems are capable of building parts from functional composite materials, typically using polymers loaded with particles or short-fibres. One of the ME systems most commonly used for composite part production is the range of machines from the Markforged company. Different machines in the range can be used with a short-fibre reinforced polyamide material and with a polymer material loaded with metal powders. The former is used to produce stiffer components and the latter to produce green parts that can then be sintered

* Corresponding author: n.kaill@,lboro.ac.uk 
into near fully dense metal parts. Some of the Markforged machines can also incorporate continuous-fibre reinforcement.

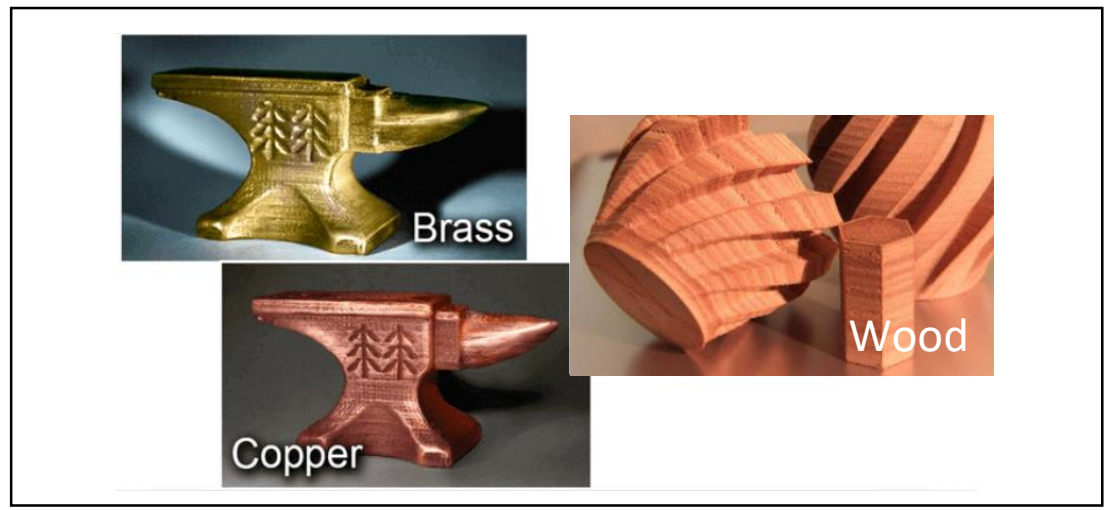

Fig. 1. ME parts made from aesthetic composite materials [1].

A limitation that is common to almost all commercial ME machines, when printing in fibre-reinforced composite material, is that the orientation of the fibres is dictated by the 2D nature of the layers that are deposited. That is, the fibre orientation can be controlled in the $\mathrm{XY}$-plane but not in the Z-direction. This is adequate for components that have loading conditions that are largely two-dimensional. However, with more complex, threedimensional loading, there is a requirement for fibre orientation in all three directions. This can be achieved by using an ME system with 5-axis capability, such as the 5AxisMaker machine offered by 5AxisMaker [2]. The work reported in this paper exploited the 5-axis capability of this machine to build a highly curved geometry represented by a simple thinwalled dome. Due to its topology, this geometry is appropriate to explore how to optimise the mechanical performance of ME parts by exploiting 5-axis capability to selectively orient the deposition of filament. The objective of the work was to determine what impact the addition of appropriately oriented short carbon fibres (SCF) would have on the strength and stiffness of dome samples made from polylactic acid (PLA) material. The work was undertaken to support the Directional Composites through Innovative Manufacturing (DiCoMI) project, funded by the European Commission.

\section{Method}

A finite element analysis (FEA) simulation was conducted to determine the direction of the stress vectors within the geometry of the dome, when loaded in compression in the direction of its central axis, i.e. the vertical axis drawn through the "pole" of the dome. The simulation was carried out within the Solidworks CAD software [3]. A quarter section of the dome's geometry was used in the simulation, with the boundary surfaces being constrained to allow sliding. A force was then applied to the top of the section, to induce the resultant stress vectors. The simulation software allows the three sets of principle stress vectors to be calculated and shown. The simulation indicated that the vertical direction is the dominant direction of the principle stress vectors. The horizontal stress component was indicated to be the next dominant component. In Figure 2 the colour of the vectors denotes the magnitude of the stress; in Solidworks, red is used to indicate high values, while green indicates a lower value. The third component of principle stress vectors (aligned perpendicularly from the inside to the outside of the dome) were negligible in comparison to the first two. This information was used to create printing strategies for the dome samples, since the direction of these stress vectors indicates the preferred orientation of the 
carbon fibres. Two sets of printer paths were needed, paths going "vertically" from the "equator" towards the "pole" of the dome (referred to as vertical printing strategy), and "horizontal" paths following the "lines of latitude" of the dome (referred to as horizontal printing strategy.
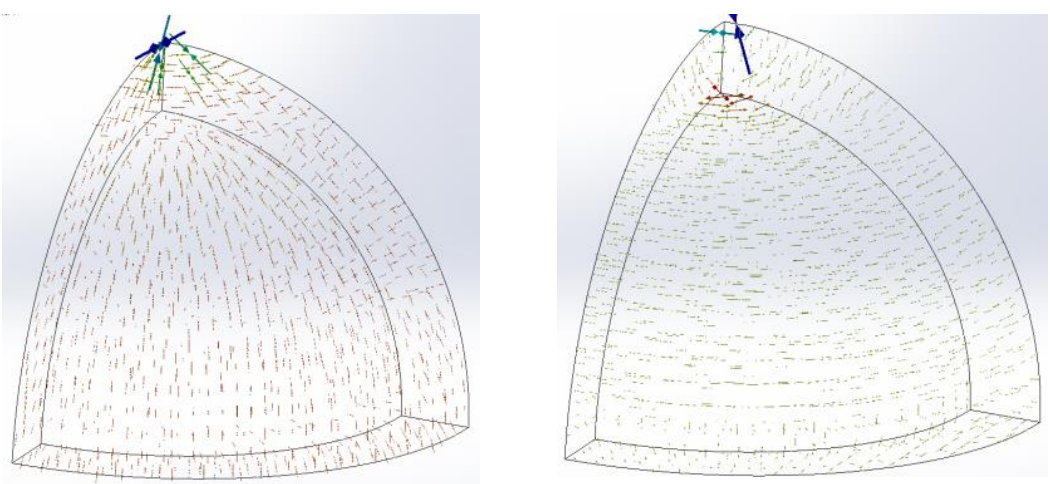

Fig. 2. Simplified FEA of Dome Section: (left) Vertical Stress Vectors, (right) Horizontal Stress Vectors.

The process of creating a 5-axis sample is shown in Figure 3 and is fully explained in a previous paper [4]. Five dome samples at each of six different conditions were built:

(i) Pure PLA material using horizontal printing strategy only (5H PLA)

(ii) Pure PLA material using vertical printing strategy only (5V PLA)

(iii) Pure PLA material using combined horizontal and vertical printing strategies (5C PLA)

(iv) SCF-reinforced PLA material using horizontal printing strategy only (5H CF)

(v) SCF-reinforced PLA material using vertical printing strategy only (5V CF)

(vi) SCF-reinforced PLA material using combined horizontal and vertical printing strategies $(5 \mathrm{C} \mathrm{CF})$

All samples were built on the same machine with printing parameters adjusted for the two different materials. The pure PLA material was sourced from Any Cubic [5] and the SCF-reinforced PLA from Technology Outlet [6]. The fibre content level was quoted as $20 \%$. Both materials were in the form of $1.75 \mathrm{~mm}$ filament.

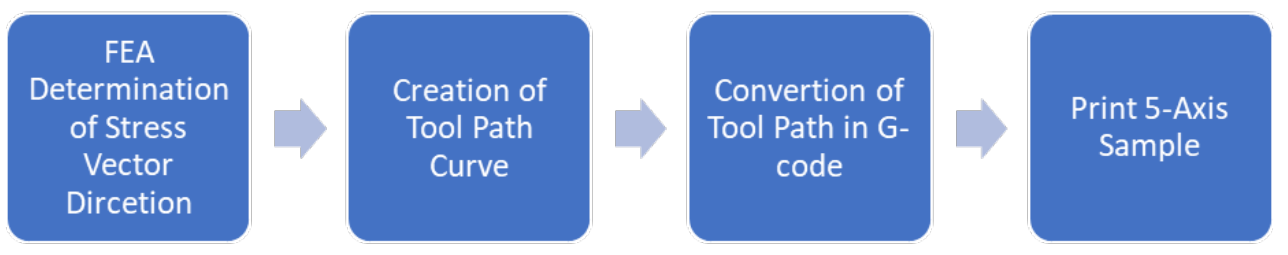

Fig. 3. Flow Chart of 5-Axis Print Process.

After the samples were built, axial compression testing was carried out on an Instron 3366 material test system with a $5 \mathrm{kN}$ load cell and a compression speed of $2 \mathrm{~mm}$.s -1 . The purpose of this test procedure was to establish the different mechanical behaviours of the 
test samples in regard to stiffness, ductile behaviour and maximum compressive loading.

The experimental setup for the testing is illustrated in Figure 4.

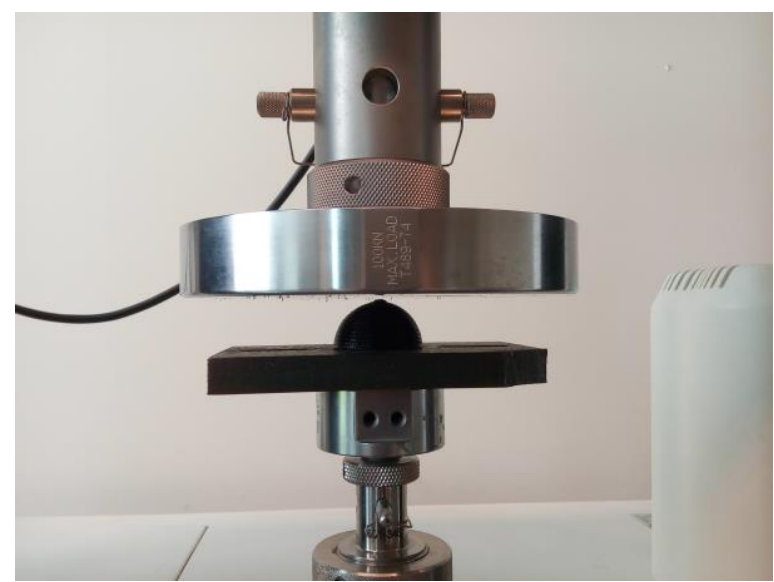

Fig. 4. Compression testing set-up.

\section{Results and discussion}

The data from the compression testing of each sample was tabulated into an Excel spreadsheet. The mean average for each set of five samples is displayed in Figure 5.

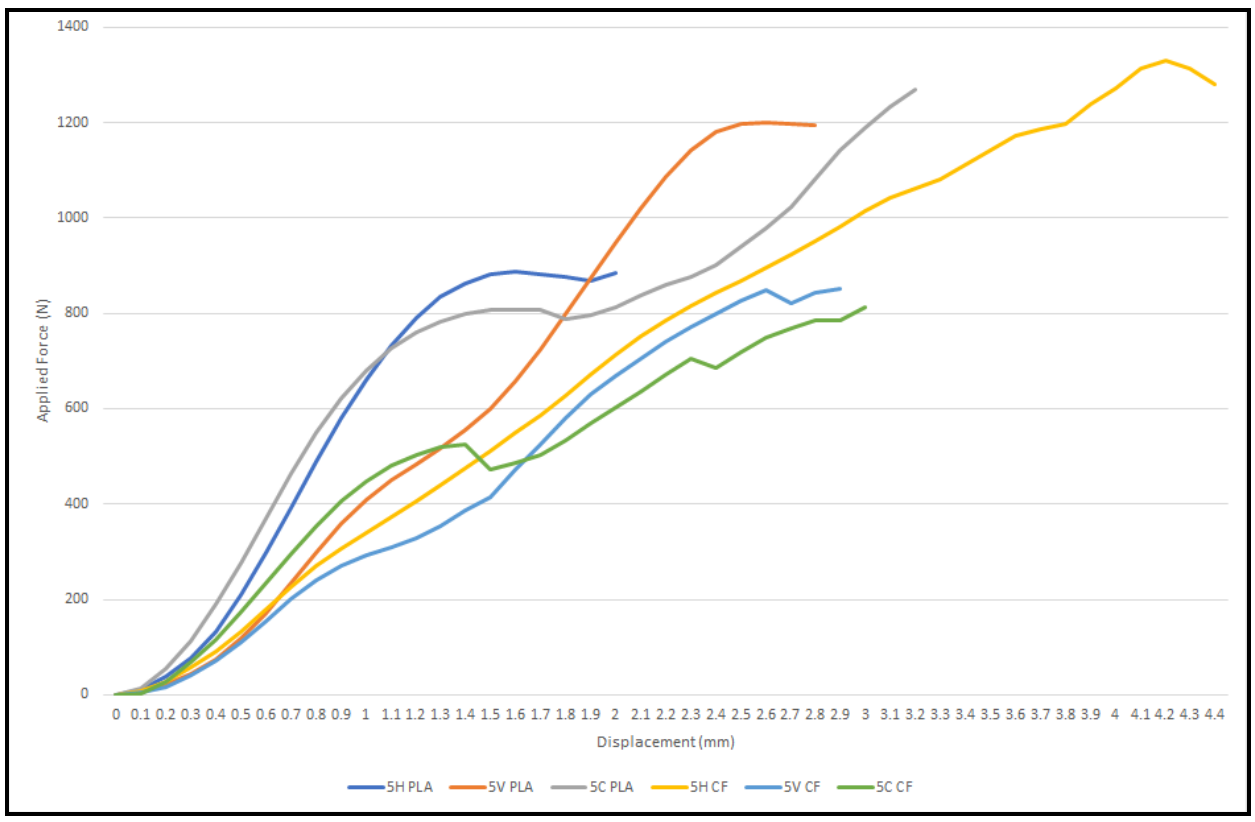

Fig. 5. Compression test results.

The results can be interpreted in two ways. Firstly, the difference in mechanical behaviours caused by the different printing strategies can be analysed separately for each of the two different materials. Here we see that, for pure PLA, the use of a combined printing strategy increases both the load-carrying capability and ductility of the domes. However, the improvement over the vertical printing strategy samples is minimal. For the SCF- 
reinforced samples, however, the horizontal printing strategy samples performed significantly better than either the vertical or combined strategy samples. This was quite unexpected and required further investigation. Detailed observation of both the vertical and combined strategy samples after testing indicated that they all failed in a very specific manner, i.e. along a vertical "tear" from the pole to the equator of the dome (see Figure 6). The authors hypothesise that the increased stiffness of the vertical print lines causes an increase in the hoop-stress within the domes. Since there is no horizontal SCF reinforcement in the vertical printing strategy domes, this leads to early failure. In the combined printing strategy dome, the horizontal SCF reinforcement should prevent this from happening. However, the nature of the horizontal printing results in a vertical "seam" that runs from the pole to the equator. At this seam, the print head reverses and goes back on itself for a further $360^{\circ}$ deposition. This is then repeated, layer upon layer. Therefore, along the whole length of this seam there is a break in the SCF reinforcement, leading to a weakness in the dome. This could be overcome by developing a "spiral" printing strategy that would avoid the creation of the seam. However, this is not feasible in the current design of 5-axis machine.

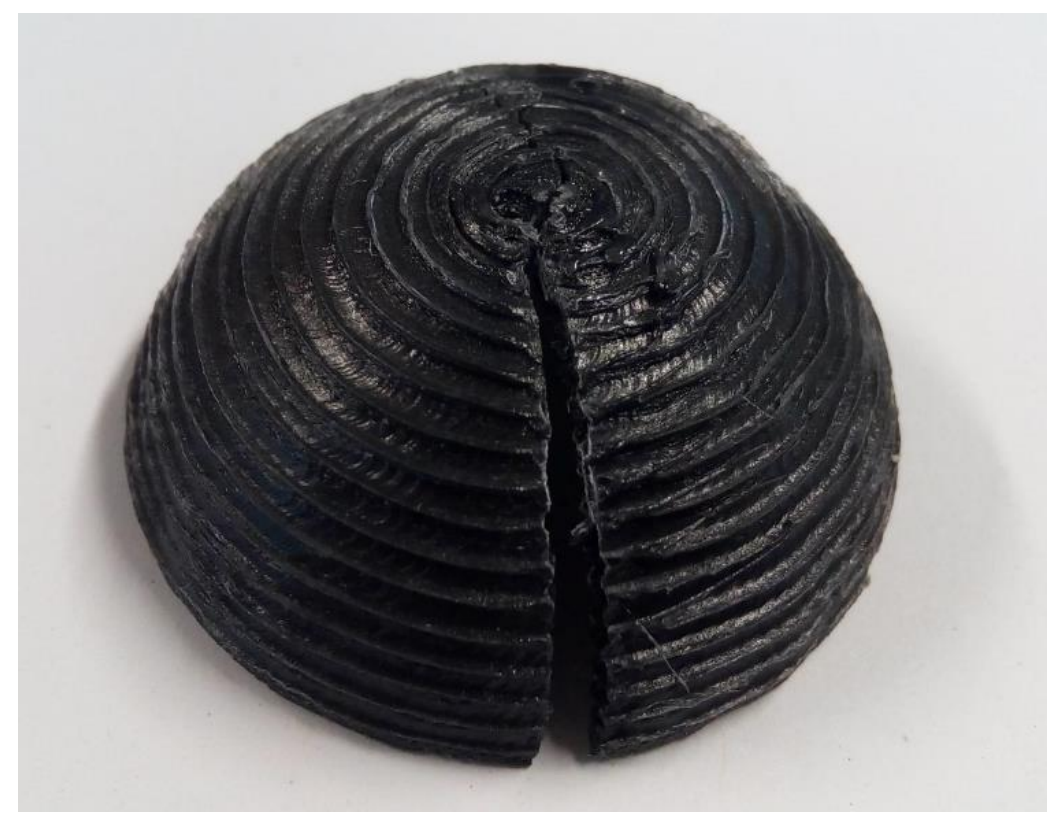

Fig. 6. Failure mechanism for the vertical and combined strategy SCF-reinforced domes.

The second way of interpreting the results of this study is to make a comparison between the two different materials, for each of the three different printing strategies. The authors had expected SCF reinforcement to increase the mechanical strength of the domes for all three printing strategies. However, the results show that only the horizontal printing strategy domes displayed this improvement. The SCF-reinforced domes were able to withstand $50.6 \%$ more force, on average, than their pure PLA counterparts. Furthermore, they also deformed over a much greater displacement before the samples failed. Similar improvements in strength were not seen for either the vertical or combined printing strategies. Instead, there was a decrease in strength and similar deformations before failure. The authors believe this phenomenon is also explained by the "early failure" explained above. Never-the-less, the improvement in strength for the horizontal strategy samples is promising and justifies further exploration of directional SCF reinforcement in 3D printing. 


\section{Conclusions and future work}

This paper has shown that the addition of short carbon fibre reinforcement to PLA parts produced on a 5 -axis $3 \mathrm{D}$ printer causes a change in the mechanical behaviour of the parts. Under certain conditions, it was possible to increase the compressive load resistance of dome-shaped parts by over $50 \%$, with an even greater increase in deformation prior to failure. However, other conditions did not show the same result indicating that the impact of SCF reinforcement is not straight-forward and required further investigation. Online references will be linked to their original source, only if possible. Future work within the DiCoMI project will investigate the use of alternative 5-axis printing strategies and different combinations of fibre and polymer matrix, including bio-composites. The use of continuous fibre reinforcement is also being investigated.

The DiCoMI project has received funding from the European Union's Horizon 2020 research and innovation programme under grant agreement No 778068 .

\section{References}

1. all3dp.com/1/3d-printer-filament-types-3d-printing-3d-filament (accessed July 2019)

2. www.5axismaker.com (accessed July 2019)

3. Solidworks CAD Software, DASSAULT Systems. www.solidworks.com

4. N. Kaill, R.I. Campbell, P. Pradel, G. Bingham, A Comparative Study Between 3-Axis and 5-Axis Additively Manufactured Samples and their Ability to Resist Compressive Loading, Proceedings of the Solid Freeform Fabrication Symposium, Austin, Texas, August 2019.

5. www.amazon.co.uk/gp/product/B07DMF6J4M/ref=ppx_yo_dt_b_asin_title_o06_s00?i $\mathrm{e}=\mathrm{UTF} 8 \& \mathrm{psc}=1$ (accessed August 2019)

6. technologyoutlet.co.uk/products/carbon-pla-3d-printer-filament (accessed August 2019) 